\title{
Research on Informationized Equipment Materials Support Mode Programming
}

\author{
Tiening WANG ${ }^{1, a}$, Yaqi DONG ${ }^{1, a}$, Yu CAO ${ }^{2, \text { a }}$ \\ ${ }^{1}$ Department of technique Support Engineering, Academy of Armored Forces Engineering, Beijing \\ 100072, China \\ ${ }^{2}$ Department of Equipment Command and Management, Academy of Armored Forces Engineering, \\ Beijing 100072, China \\ aemail: changxiangtianhao@163.com
}

Keywords: informationize; equipment materials support; Petri-net

\begin{abstract}
The existing equipment materials support mode has many shortages under the new situation in the aspects of resources configure, inter-profession cooperation, information sharing, etc. Therefore, a new support mode, an acquisition and supply information system and the corresponding supporting process was planned, and the model of the supporting process was then built based on Petri-net.
\end{abstract}

\section{Introduction}

With the trend of combined joint warfare and unified equipment support mode, as well as the increasing military application of information technology, latest weapons are equipped in a large scale, and more drills are held, causing the increase of equipment damage rate. Therefore, how to use the information and computer technology to achieve a new support mode to integrate the resource, which is featured with exact and demand-driven, is now a significant and urgent task.

\section{Problems of existing equipment materials support mode}

The organization of existing equipment materials support mode is based on the equipment profession, thus the support work is managed by multi-departments. With the military informationization, such storage-leading support mode has exposed a load of problems, making it hardly to achieve the goal of unified equipment support.

(1) The management of equipment materials is divided in several departments by profession. They are similar in support procedure but isolated with each other in business, causing waste of support resources and lack of inter-profession cooperation.

(2) Mainly depending on the staff's experience, inventory structure is Unreasonable. The basic troops who need the materials most, however, lack equipment materials. Once facing emergency, this storage strategy cannot support the demand of troops in time.

(3) Although numerous information systems have been developed, most of them are isolated because of no unified standards and lack network.

(4) The materials demand information and logistics go step-by-step, with low supply efficiency. And the information will lose its accuracy and timeliness because of the time delay and human factors.

\section{Informationized Equipment Materials Support Mode (IEMSM)}

To settle the problems the current equipment materials support mode existing, we should optimize and recombine the support elements and establish new equipment materials support mode under the information condition through taking use of the network techniques and information management techniques. Through integrated the materials and information flow, the new mode should provide timely and exact support service.

(1) Programming of IEMSM

IEMSM depicted as Figure 1 is constructed based on the Materials Acquisition and Supply 
Information System (MASIS), which will be introduced latter. The two departments, Strategic Materials Acquisition and Supply Center (S-MASC) and Local Materials Acquisition and Supply Center (L-MASC), controls the data-flow of materials support, while the global materials support center(G-MSC), local materials support center(L-MSC) and tactical materials support center(T-MSC) take charge of the materials-flow collectively, implementing combined support for all kinds of army categories and support professions.

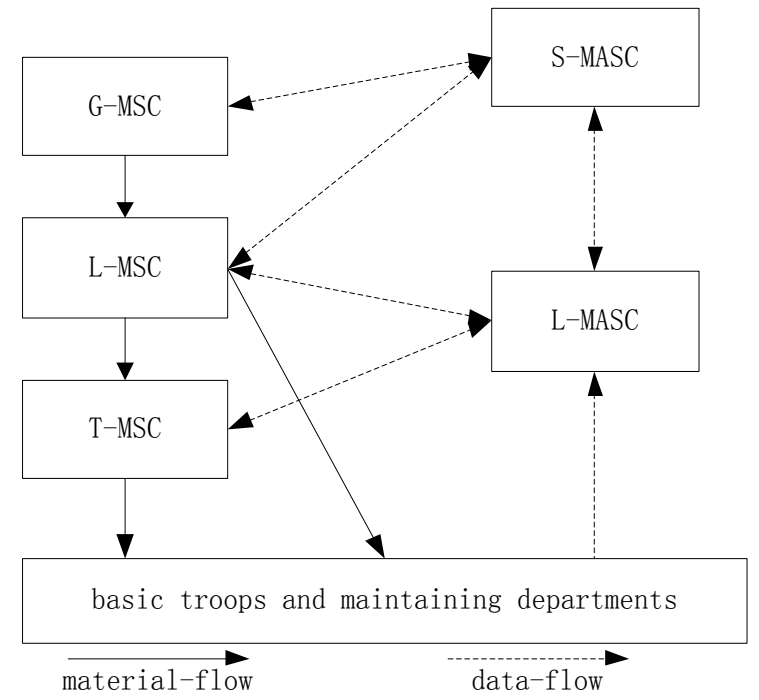

Fig.1. demonstration of IEMSM

To be more detailed, S-MASC, as an organization, consists of transactional staff from different support departments, in charge of attaining global-level materials information, making decisions for materials acquisition, storage and supply. The L-MASC, correspondingly, do the same job but in campaign level. On the left side, G-MSC, L-MSC and T-MSC work as logistics transfer station, from large scale to small. They respectively execute the commands sent by S-MASC or L-MASC.

IEMSM has following features: profession combined management reduces repetitive constructions, region divided support improves support efficiency and flexibility, information leading business boosts the transition to accurate support.

(2) Programming of MASIS

As the basis and core of IEMSM, MASIS (Materials Acquisition and Supply Information System) provides an available approach to fulfill the new support mode just mentioned. By recombining the functional components of support process, MASIS is an integrated and unified materials support logistics system, of which the S-MASC is the heart section. It can be easily seen from Figure 2 that MASIS comprises six units, which are S-MASC, Storage and Supply Logistics System (SSLS), Business Management System (BMS), Commanding and Controlling System (CCS), Emergent Support System (ESS), Integrated Database. These six units play different roles, but they are mutually connected with each other. 


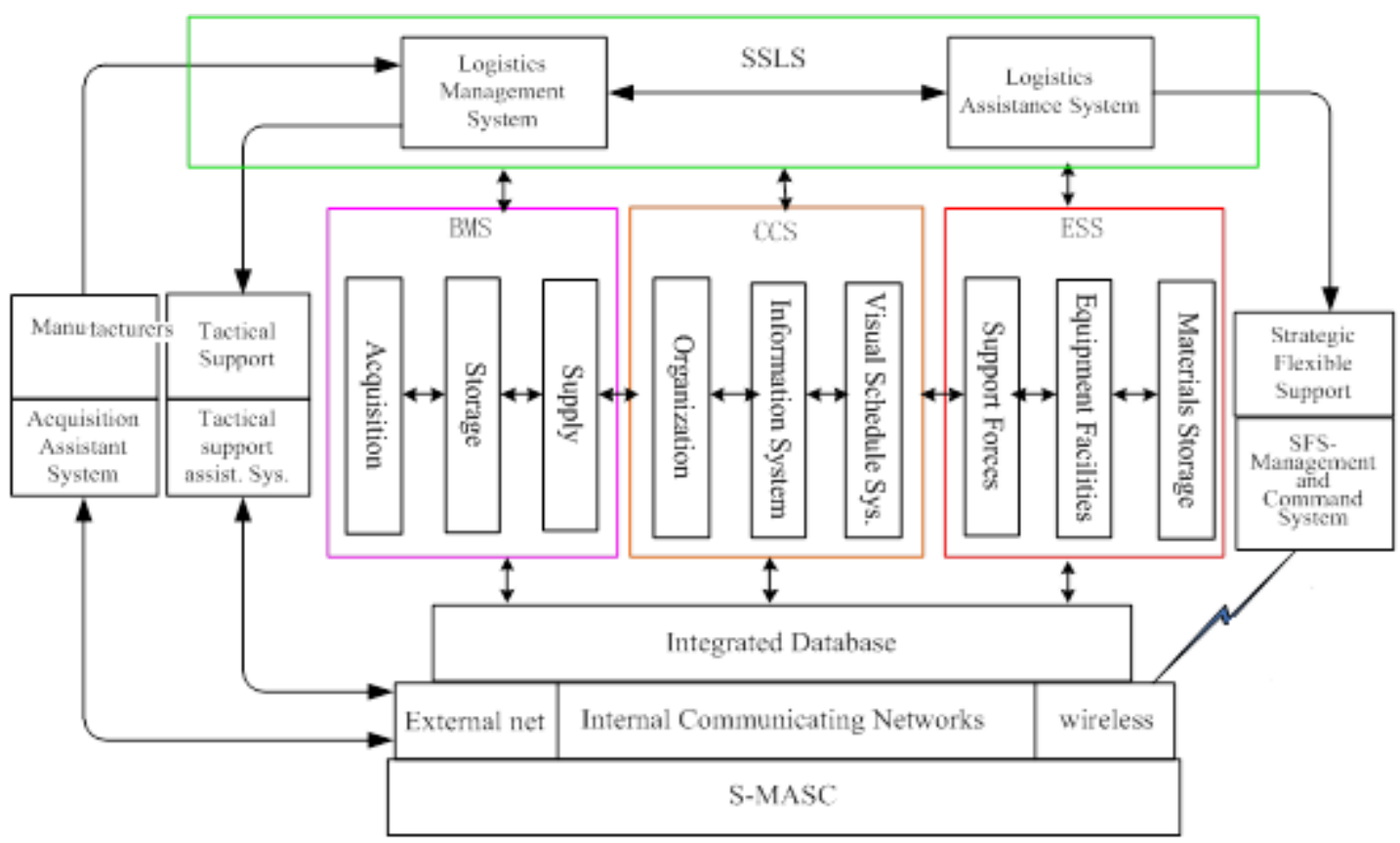

Fig.2. structure of MASIS

Among the 6 units, SSLS is in charge of the logistic job following IEMSM just mentioned, with both military and civilian logistic resources; the job BMS is managing the acquisition, storage and supply business and providing decision-making assistance; CCS can monitor and schedule the materials by Visual Schedule System; ESS mainly completes the emergent support job. By integrating the 6 units, MASIS helps the army build a combined and unified materials support process, with materials transmitted efficiently and data flowing fluently.

\section{Process Modeling of IEMSM based on Petri-networks}

Building the model of IEMSM process can be helpful for latter possible simulation. Petri-networks is a kind of simple but combined modeling method, and it can be used both briefly and easy-understanding to imitate the discrete incident system. So hierarchical Petri-networks is chosen to build the support process model of IEMSM as Figure 3.

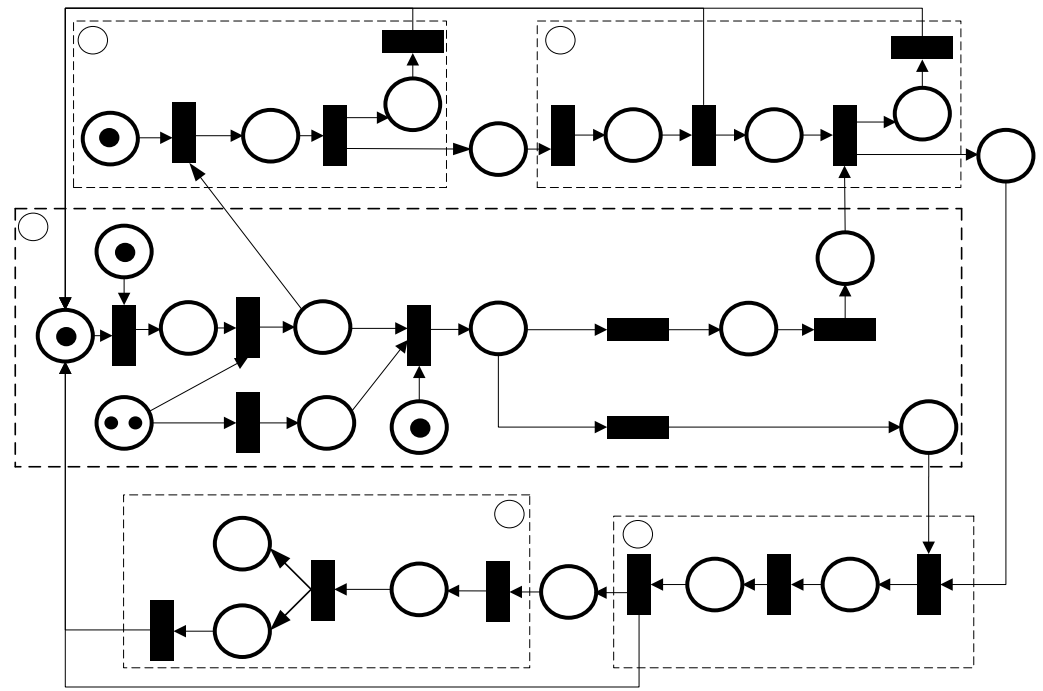

Fig.3. IEMSM materials support model based on Petri-networks

In the figure above, (1) (5) represents the unit of acquisition, manufacturing, supply, storage, consumption and decision-making respectively. The meaning of the places and transitions in figure 3 are listed in the following table. 
Table1. meaning of transitions and places of IEMSM process model

\begin{tabular}{|c|c|c|c|}
\hline transitions & meaning & Places & meaning \\
\hline T01 & Enquire materials consumption & P03 & Consumption and inventory info. \\
\hline T02 & Acquisition decision-making & P04 & Acquisition plan \\
\hline T03 & Demand forecasting & P05 & Materials demand \\
\hline T04 & Distribution decision-making & P06 & Fund limitation \\
\hline T05 & Supply decision-making & P07 & Distribution plan \\
\hline T06 & Assign distributing plan & P08 & Transport and supply scheme \\
\hline T07 & Assign supply scheme & P09 & Transport and supply task \\
\hline T11 & Choose supply source & P010 & Quantity of materials acquired \\
\hline $\mathrm{T} 12$ & Sign contract & P11 & Supplier alternatives \\
\hline T13 & Report contract & $\mathrm{P} 12$ & Supplier selection \\
\hline $\mathrm{T} 21$ & Make manufacture plan & P13 & Reservation and contract \\
\hline $\mathrm{T} 22$ & manufacturing & $\mathrm{P} 1$ & Interface, acquisition completed \\
\hline T23 & Transport and supply & P21 & Manufacture plan \\
\hline T24 & Report supply implement & P22 & Manufacture state \\
\hline T31 & Receive materials & P23 & Supply state \\
\hline T32 & Check in materials & $\mathrm{P} 2$ & Interface, supply completed \\
\hline T33 & Update inventory & P31 & Materials received \\
\hline T41 & Materials application & P32 & Checked in \\
\hline T42 & Materials consumption & P3 & Interface, checked in \\
\hline T43 & Report consumption & P41 & Ready to consumption \\
\hline $\mathrm{P} 0$ & Ready to proceed & P42 & Consumption information \\
\hline $\mathrm{P} 01$ & Information center & P43 & end \\
\hline P02 & Maintaining and consumption info. & & \\
\hline
\end{tabular}

\section{Conclusion}

In order to solve the problems of existing equipment materials support mode, a new support mode called IEMSM is proposed, and a new integrated information system called MASIS, which is the basis of IEMSM, is programmed, the support process model of IEMSM is built for latter simulation and test. All of these jobs provide an possible scheme for the develop of unified equipment support.

\section{References}

[1] ZHANG Wei, YU Chunfeng. Research on Equipment Integrated Synthetic Support in Joint Operations[J]. Journal of the Academy of Equipment Command \& Technology, 2011, 22(2):1- 4.

[2] LIANG Haibing. Unified Equipment Support [M]. Liberation Army Press, 2009.

[3] LIU Hongshui. Research On Simulation Model Of Armored Equipment Materiel Support Logistics System [D]. AAFE, 2011.

[4] LIANG Bo. Research On Design And Survivability Of Universal Equipment Materiel Support Network [D]. AAFE, 2012.

[5] CAO Yu. Research on Equipment Material Support Based on Acquisition and Supply Center [J]. AAFE, 2009, 23(3): 11-14. 